\title{
AN APPLICATION OF AN OPTIMAL BEHAVIOUR OF THE GREEDY SOLUTION IN NUMBER THEORY
}

\author{
B. VIZVÁRI (Ankara)
}

\begin{abstract}
Let $a_{1}, a_{2}, \ldots, a_{n}$ be relative prime positive integers. The Frobenius problem is to determine the greatest integer not belonging to the set $\left\{\sum_{j=1}^{n} a_{j} x_{j}: x \in\right.$ $\left.Z_{+}^{n}\right\}$. The Frobenius problem belongs to the combinatorial number theory, which is very rich in methods. In this paper the Frobenius problem is handled by integer programming which is a new tool in this field. Some new upper bounds and exact solutions of subproblems are provided. A lot of earlier results obtained with very different methods can be discussed in a unified way.
\end{abstract}

\section{Introduction}

For given positive integers $a_{1}, \ldots, a_{n}$ the set of representable integers is defined as

$$
F=\left\{\sum_{j=1}^{n} a_{j} x_{j}: x \in Z_{+}^{n}\right\} .
$$

If $b \in F$ and

$$
\sum_{j=1}^{n} a_{j} x_{j}=b
$$

holds with $x \in Z_{+}^{n}$, then the vector $\mathbf{x}$ is called the representation of $b$. It is wellknown that if

$$
\text { g.c.d. }\left(a_{1}, \ldots, a_{n}\right)=1
$$

then $F$ contains all of the integers above a threshold. Throughout this paper it is always assumed that ( 1 ) is satisfied. The greatest integer not belonging to $F$, i.e. the smallest possible threshold, is denoted by

$$
g\left(a_{1}, \ldots, a_{n}\right) \text {. }
$$

Mathematics subject classification numbers, 1991. Primary 11A99, 11 B99.

Key words and phrases. Frobenius problem, Greedy solution, integer programing. 
The Frobenius problem of number theory is to determine this number, called Frobenius number. This problem belongs to the so-called combinatorial number theory which is very rich in methods. The aim of this paper is to show that the problem can be attacked with integer programming, as well. Although many former results are discussed here, the proofs are quite different, and in most of the cases they are simpler. Furthermore all of them are obtained by the same technique of proof.

Although [Kannan 92] showed that for fixed $n$ the Frobenius problem is a polynomially solvable with a complicated algorithm, there is an opinion among the experts of the field that there is no hope for a general formula giving the Frobenius number. Thus the closed formulas of special cases have a great interest. These kind of results are presented in Section 6. They are based on some upper bounds of a special class of the Frobenius problem discussed in Section 5. A way of the extending of the results is elaborated in Section 7. The results of this paper are achieved with the use of three tools. The first one is an integer programming reformulation of the Frobenius problem. It was proposed in [Vizvári 84] and [Vizvári 87]. The other two tools are the greedy method of the knapsack problem and its optimal behaviour. These are discussed in Section 4. Finally the special class of the Frobenius problem is defined in Section 3.

\section{An equivalent form of the Frobenius problem}

In [Brauer-Shockley 62] the following has been proved.

THEOREM 1. Let $k$ be an arbitrary index. Denote $M_{r}$ the residue class $\bmod a_{k}$ containing $r\left(1 \leq r<a_{k}\right)$. Let the integer $t_{r}$ be defined by

$$
t_{r}=\min \left\{b \in F \cap M_{r}\right\} \text {. }
$$

Then

$$
g\left(a_{1}, \ldots, a_{n}\right)=\max \left\{t_{r}: 1 \leq r<a_{k}\right\}-a_{k} .
$$

The following reformulation of the Frobenius problem is based on this statement. If for an index $j$ the equation $a_{j}=1$ holds then $F=Z_{+}$and thus $g\left(a_{1}, \ldots, a_{n}\right)=-1$, which is an uninteresting case. Throughout the entire paper we may assume without loss of generality that $a_{1}$ is the minimal among the $a_{j}$ 's, i.e.

$$
1<a_{1}<a_{2}, \ldots, a_{n} .
$$

Theorem 1 will be applied with $k=1$. To do this the positive integers $\alpha_{j}, \beta_{j}$ $j=2, \ldots, n$ are defined as follows

$$
a_{j}=\alpha_{j} a_{1}+\beta_{j} \quad \text { and } \quad 0<\beta_{j}<a_{1} .
$$

The case $\beta_{j}=0$ is excluded here, because in that case the number $a_{j}$ is superfluous, i.e. without changing the Frobenius number $a_{j}$ can be eliminated from the problem. 
The optimal value of the knapsack problem

$$
\begin{gathered}
\min \left(x_{1}+\sum_{j=2}^{n} \alpha_{j} x_{j}\right) \\
-a_{1} x_{1}+\sum_{j=2}^{n} \beta_{j} x_{j}=b \\
x \in Z_{+}^{n}
\end{gathered}
$$

is denoted by $z(b)$. The subproblem obtained from $(K(b))$ fixing the variable $x_{1}$ at zero is denoted by $K\left(b \mid x_{1}=0\right)$ and its optimal value is $z\left(b \mid x_{1}=0\right)$.

TheOREM 2. [Vizvári 87] Using the above notations

$$
t_{r}=a_{1} z(r)+r
$$

and

$$
g\left(a_{1}, \ldots, a_{n}\right)=\max _{1 \leq r<a_{1}}\left\{a_{1} z(r)+r\right\}-a_{1}
$$

hold.

\section{A class of the Frobenius problem}

In this paper only the problems satisfying the conditions

$$
\begin{aligned}
\beta_{2} & =1, \\
\frac{\alpha_{j}}{\beta_{j}} \geq \frac{\alpha_{j+1}}{\beta_{j+1}} j & =2, \ldots, n-1, \\
\beta_{j}<\beta_{j+1} j & =2, \ldots, n-1
\end{aligned}
$$

are considered. Many special cases solved exactly in the literature belong to that class, e.g. [Roberts 56], [Dulmage-Mendelsohn 64], [Byrnes 74], [Siering 74], [Selmer 77]. Some of these results are discussed in Section 6, because they are special cases of Theorems 10 and 13 of this paper.

\section{The greedy method}

Let $\left\{c_{n}\right\}$ and $\left\{d_{n}\right\}$ be two infinite sequences of positive integers. The knapsack problem

$$
\begin{gathered}
f(n, b)=\min \sum_{j=1}^{n} c_{j} x_{j} \\
\sum_{j=1}^{n} d_{j} x_{j}=b \\
x \in Z_{+}^{n}
\end{gathered}
$$


has two parameters, the right-hand side and the number of variables. (This the reason that the optimal value is considered as the function of these two quantities.) The greedy solution of the problem is defined by the equation

$$
x_{j}^{g}=\left\lfloor\frac{b-\sum_{k=j+1}^{n} d_{k} x_{k}^{g}}{d_{j}}\right\rfloor \quad j=n, n-1, \ldots, 1
$$

It is obvious that the greedy solution is feasible for all $n$ and $b$ if and only if $d_{1}=1$. The value of the greedy solution is a function of the number of variables and the right-hand side and it is

$$
h(n, b)=\sum_{j=1}^{n} c_{j} x_{j}^{g} .
$$

The following two conditions will be referred in several statements

$$
\begin{gathered}
d_{1}<d_{2}<\ldots \\
d_{1}=1 \\
\frac{c_{j}}{d_{j}} \geq \frac{c_{j+1}}{d_{j+1}}, \quad j=1,2, \ldots
\end{gathered}
$$

THEOREM 3. [Magazine et al. 75] The integers $m$ and $v$ are defined by the constraints $d_{n+1}=m d_{n}-v, 0 \leq v<d_{n}$. Assume that the Conditions (12) and (19) and (14) are satisfied and for a fixed $n$ the one-variable functions $f(n,$.$) and$ $h(n,$.$) are identical. Then the following three statements are equivalent.$

(a) The one-variable functions $f(n+1,$.$) and h(n+1,$.$) are identical,$

(b) $f\left(n+1, m d_{n}\right)=h\left(n+1, m d_{n}\right)$,

(c) $c_{n+1}+h(n, v) \leq m c_{n}$.

Theorem 4. If (12) and (19) and (14) hold and the numbers $\frac{d_{j+1}}{d_{j}}(j=$ $1, \ldots, n-1)$ are integer, then the one-variable functions $f(p,$.$) and h(p,).(p=$ $1, \ldots, n)$ are identical.

Proof. Obviously $f(1,.) \equiv h(1,$.$) , because in this case \forall b$ the knapsack problem has only one feasible solution. Assume that the statement has been proven for $p<n$. Then the last theorem can be applied with $m=\frac{d_{p+1}}{d_{p}}$ and $v=0$. Hence $h(n, v)=0$. Thus Constraint (c) becomes

$$
c_{p+1} \leq \frac{d_{p+1}}{d_{p}} c_{p}
$$

which follows from (14). 
THEOREM 5. [Vizvári 84] If (13) holds then the greedy solution is the only feasible solution of the following constraints

$$
\begin{aligned}
& d_{1} x_{1}+d_{2} x_{2}+\cdots+d_{n-1} x_{n-1}+d_{n} x_{n}=b \\
& d_{1} x_{1}+d_{2} x_{2}+\cdots+d_{n-1} x_{n-1} \leq d_{n}-1 \\
& d_{1} x_{1}+d_{2} x_{2} \quad \leq d_{3}-1 \\
& d_{1} x_{1} \quad \leq d_{2}-1 \\
& x \in Z_{+}^{n} \text {. }
\end{aligned}
$$

The same notation is used if the greedy method is applied for Problem $\left(K\left(b \mid x_{1}=0\right)\right)$, i.e.

$$
x_{j}^{g}=\left\lfloor\frac{b-\sum_{k=j+1}^{n} \beta_{k} x_{k}^{g}}{d_{j}}\right\rfloor \quad j=n, n-1, \ldots, 2
$$

and the value of the greedy solution is $h\left(b \mid x_{1}=0\right)$.

\section{Upper bounds}

After these preliminaries the investigation of the problem class defined in Section 2 can be started.

Let $h_{j}$ and $r_{j}(j=3, \ldots, n+1)$ be the following numbers

$$
\begin{array}{r}
h_{j}=\max \left\{h\left(r \mid x_{1}=0\right): 1 \leq r<\beta_{j}\right\}, \\
r_{j}=\max \left\{r: h\left(r \mid x_{1}=0\right)=h_{j}: 1 \leq r<\beta_{j}\right\},
\end{array}
$$

where $\beta_{n+1}=a_{1}$. The greedy solution determining $h_{n+1}$ and $r_{n+1}$ gives an upper bound for the Frobenius number in the case of an even wider subclass than that is defined in Section 2, as it is described in the next theorem. The other $h_{j}$ 's and $r_{j}$ 's are important in the calculation of $h_{n+1}$ and $r_{n+1}$.

THeOREM 6. If $n \geq 3$ and the Condition (8) is satisfied then

$$
g\left(a_{1}, \ldots, a_{n}\right) \leq a_{1}\left(h_{n+1}-1\right)+r_{n+1} .
$$

Proof. Theorem 2 gives the equation

$$
g\left(a_{1}, \ldots, a_{n}\right)=\max _{1 \leq r<a_{1}}\left\{a_{1} z(r)+r\right\}-a_{1}
$$


As obviously $z(r) \leq z\left(r \mid x_{1}=0\right)$ the inequality

$$
g\left(a_{1}, \ldots, a_{n}\right) \leq \max _{1 \leq r<a_{1}}\left\{a_{1} z\left(r \mid x_{1}=0\right)+r\right\}-a_{1}
$$

follows immediately. The right-hand side does not decrease if the optimal solution is substituted by the greedy one. The existence of the greedy solution follows from (8). Thus

$$
\max _{1 \leq r<a_{1}}\left\{a_{1} z\left(r \mid x_{1}=0\right)+r\right\} \leq \max _{1 \leq r<a_{1}}\left\{a_{1} h\left(r \mid x_{1}=0\right)+r\right\}
$$

The right-hand side is maximal if $h\left(r \mid x_{1}=0\right)$ is maximal as $1 \leq r<a_{1}$. Hence

$$
\max _{1 \leq r<a_{1}}\left\{a_{1} h\left(r \mid x_{1}=0\right)+r\right\}=a_{1} h_{n+1}+r_{n+1}
$$

and the theorem follows.

The upper bound given in the theorem is denoted by $\psi$, i.e.

$$
\psi=a_{1}\left(h_{n+1}-1\right)+r_{n+1} .
$$

The next aim is to show that $\psi$ can be determined with $O\left(n^{2}\right)$ operations. To do that the following theorem is needed.

THEOREM 7. Let $w^{(j)}$ and $u^{(j)}$, resp., be the greedy solutions of the problems $\left(K\left(r_{j} \mid x_{1}=0\right)\right)$ and $\left(K\left(\beta_{j}-1 \mid x_{1}=0\right)\right)(j=3, \ldots, n)$, resp. Then either $w^{(j)}=u^{(j)}$ (in the case of $r_{j}=\beta_{j}-1$ ) or there is an index $k(3 \leq k<j$ ) such that

$$
\begin{aligned}
w_{i}^{(j)} & =u_{i}^{(j)} & & i=k+1, \ldots, n \\
w_{k}^{(j)} & =u_{k}^{(j)}-1 & & \\
w_{i}^{(j)} & =w_{i}^{(j)} & i & =2, \ldots, k-1 .
\end{aligned}
$$

Proof. Assume $r_{j}<\beta_{j}-1$. Let $k$ be the greatest index such that

$$
w_{k}^{(j)} \neq u_{k}^{(j)} \text {. }
$$

As $r_{j}<\beta_{j}-1$ it follows from the greedy property that $w_{k}^{(j)}<u_{k}^{(j)}$. If $k=2$ then

$$
\sum_{i=2}^{n} \alpha_{i} w_{i}^{(j)}-\sum_{i=2}^{n} \alpha_{i} u_{i}^{(j)}=\alpha_{2}\left(w_{2}^{(j)}-\alpha_{2} u_{2}^{(j)}\right)<0
$$

holds which contradicts the definition of $r_{j}$. Thus $k \geq 3$ and hence

$$
\beta_{j}-1=\sum_{i=2}^{j-1} \alpha_{i} u_{i}^{(j)} \geq \sum_{i=k+1}^{j-1} \alpha_{i} u_{i}^{(j)}+\beta_{k} w_{k}^{(j)}+\beta_{k} .
$$

The range of the possible values of

$$
\sum_{i=2}^{k-1} \beta_{i} w_{i}^{(j)}
$$


contains all integers in the interval $\left[0, \beta_{k}-1\right]$ regardless to the value of $w_{k}^{(j)}$, therefore both of them must be as great as possible regarding to the objective function, i.e.

$$
\begin{aligned}
& w_{k}^{(j)}=u_{k}^{(j)}-1 \\
& \sum_{i=2}^{k-1} \beta_{i} w_{i}^{(j)}=r_{k}
\end{aligned}
$$

THEOREM 8. The value of $\psi$ can be determined with $O\left(n^{2}\right)$ operations.

Proof. It is enough to show that the statement holds for determination of all of the numbers $h_{j}$. The equation $r_{3}=\beta_{3}-1$ follows immediately. Assume that the numbers $r_{i}(i=3, \ldots, j-1)$ are known. Then according to the last theorem the equation

$$
r_{j}=\sum_{i=2}^{n} \beta_{i} w_{i}^{(j)}=\sum_{i=k}^{j-1} \beta_{i} u_{i}^{(j)}-\beta_{k}+r_{k}
$$

holds for an appropriate index $k$. Thus

$$
h_{j}=\sum_{i=2}^{n} \alpha_{i} w_{i}^{(j)}=\sum_{i=k}^{j-1} \alpha_{i} u_{i}^{(j)}-\alpha_{k}+h_{k}
$$

Here $u_{i}^{(j)}=0(i=j, \ldots, n)$. Let $h_{2}=\alpha_{2}$. Then

$$
h_{j}=\max \left\{\sum_{i=k}^{j-1} \alpha_{i} u_{i}^{(j)}-\alpha_{k}+h_{k}: 2 \leq k<j\right\} .
$$

To obtain the vector $u^{(j)}$ the greedy method has to be applied which is $O(n)$ operations. Then to evaluate the right-hand side of $(20)$ the maximum is determined which needs $O(j)=O(n)$ operations. This procedure has to be executed for $j=4, \ldots, n$, thus the statement follows.

ThEOREM 9. If $n \geq 3$ and the Conditions (8)-(10) are satisfied, then the inequality

$$
g\left(a_{1}, \ldots, a_{n}\right) \leq \sum_{j=2}^{n} a_{j} \frac{\beta_{j+1}}{\beta j}-\sum_{j=1}^{n} a_{j}
$$

holds, where $\beta_{n+1}=a_{1}$.

Proof. It follows from Theorem 5 that the only feasible solution of the prob- 
lem

$$
\begin{array}{ll}
\beta_{2} x_{2}+\beta_{3} x_{3}+\cdots+\beta_{n-1} x_{n-1}+\beta_{n} x_{n} & =r \\
\beta_{2} x_{2}+\beta_{3} x_{3}+\cdots+\beta_{n-1} x_{n-1} & \leq \beta_{n}-1 \\
\quad \cdots & \leq \beta_{4}-1 \\
\beta_{2} x_{2}+\beta_{3} x_{3} & \leq \beta_{3}-1 \\
\beta_{2} x_{2} & \\
x_{j} \in Z_{+} & \\
\max \left(\alpha_{2} x_{2}+\alpha_{3} x_{3}+\cdots+\alpha_{n-1} x_{n-1}+\alpha_{n} x_{n}\right) &
\end{array}
$$

is the greedy solution. Thus the optimal value of the objective function is $h\left(r \mid x_{1}=\right.$ $0)$. An upper bound of this value is obtained if the integrality conditions are dropped and only $x \in R_{+}^{n}$ is claimed. It follows from (9) that in the optimal continuous solution the variables are in the index order as great as possible. Let $k$ be the index, such that $\beta_{k-1} \leq r<\beta_{k}$ holds. It follows from (8) that $k$ exists. Then the optimal solution of the relaxed problem is

$$
\begin{gathered}
x_{j}=\frac{\beta_{j+1}}{\beta j}-1 \quad j=2, \ldots, k-1 \\
x_{k}=\frac{r+1}{\beta_{k}}-1 \\
x_{j}=0 \quad j=k+1, \ldots, n .
\end{gathered}
$$

Hence

$$
h\left(r \mid x_{1}=0\right) \leq \sum_{j=2}^{k-1} \alpha_{j}\left(\frac{\beta_{j+1}}{\beta j}-1\right)+\alpha_{k}\left(\frac{r+1}{\beta k}-1\right) .
$$

Here the right-hand side is an increasing function of $r$, thus it is maximal at $r=$ $a_{1}-1$. Thus

$$
h_{n+1}=\max \left\{h\left(r \mid x_{1}=0\right): 1 \leq r<a_{1}\right\} \leq \sum_{j=2}^{n} \alpha_{j} \frac{\beta_{j+1}}{\beta j}-\sum_{j=2}^{n} \alpha_{j}
$$

where $\beta_{n+1}=a_{1}$. It follows from Theorem 6 that

$$
g\left(a_{1}, \ldots, a_{n}\right) \leq a_{1}\left(\sum_{j=2}^{n} \alpha_{j} \frac{\beta_{j+1}}{\beta j}-\sum_{j=2}^{n} \alpha_{j}-1\right)+a_{1}-1=\sum_{j=2}^{n} \alpha_{j} \frac{\beta_{j+1}}{\beta j}-\sum_{j=1}^{n} \alpha_{j}
$$

Similarly to the previous upper bound the following notation is introduced.

$$
\phi=\sum_{j=2}^{n} \alpha_{j} \frac{\beta_{j+1}}{\beta j}-\sum_{j=1}^{n} a_{j}
$$




\section{When give the upper bounds the exact value?}

In this section it is shown that the upper bounds are sharp in the sense that there are subclasses of the problem where they coincide with the Frobenius problem.

Theorem 10. If the Conditions (8)-(10) are satisfied and the numbers

$$
\frac{\beta_{j+1}}{\beta j} \quad j=2, \ldots, n
$$

are integers (where $\beta_{n+1}=a_{1}$ ), then the Frobenius number is $\phi$.

Proof. It is enough to show that in the proof of the last theorem all of the inequalities hold with equation. For $r=a_{1}-1$ the optimal solution of the continuous relaxation of (21) is integer, therefore it is the appropriate greedy solution. Thus (25) holds with equation. According to Theorem 4 in the present case the greedy solution is optimal, i.e. $z\left(a_{1}-1 \mid x_{1}=0\right)=h\left(a_{1}-1 \mid x_{1}=0\right)$. Thus it is enough to show that the restriction $x_{1}=0$ does not increase the value of the objective function. Because $\beta_{n}$ is a divisor of $\beta_{n+1}=a_{1}$ therefore in the greedy solution of the equation

$$
\sum_{j=2}^{n} \beta_{j} x_{j}=a_{1} x_{1}+r
$$

the value of $x_{n}$ is

$$
x_{n}^{g}=\left\lfloor\frac{a_{1} x_{1}+r}{\beta_{n}}\right\rfloor=\frac{a_{1} x_{1}}{\beta_{n}}+\left\lfloor\frac{r}{\beta_{n}}\right\rfloor
$$

and thus the values of all other variables are the same as in the determination of $h\left(r \mid x_{1}=0\right)$. Thus the increase of $x_{1}$ cannot improve the solution.

[Hujter 82] proved the statement in the case $\alpha_{2}=\cdots=\alpha_{n}=1$ by congruence considerations.

THEOREM 11. If the Conditions (8)-(10) are satisfied and the numbers

$$
\frac{\beta_{j+1}}{\beta j} \quad j=2, \ldots, n-1
$$

are integers and the inequality

$$
h_{n+1} \leq h\left(r_{n+1}+k a_{1}\right)+k
$$

holds for every positive integer $k$, then $\psi$ is the appropriate Frobenius number.

Proof. The optimality of the greedy solution in $\left(K\left(b \mid x_{1}=0\right)\right)$ follows again from Theorem 4. It can be seen from (26) that there is an optimal solution of $\left(K\left(r_{n+1}\right)\right)$ with $x_{1}=0$. Hence the two upper bounds (18) and (19) in the proof of Theorem 6 hold with equation. 
TheOREM 12. Assume that the Conditions (8)-(10) are satisfied and the numbers

$$
\frac{\beta_{j+1}}{\beta j} \quad j=2, \ldots,-1
$$

are integers. Let $\mathbf{y}$ be the greedy solution of Problem $\left(K\left(a_{1} \mid x_{1}=0\right)\right)$. Then

$$
\psi=\max \left\{\sum_{j=2}^{n} a_{j}\left(\frac{\beta_{j+1}}{\beta j}-1\right)+a_{k}\left(y_{k}-1\right)+\sum_{j=k+1}^{n} a_{j} y_{j}: k=2, \ldots, n\right\} .
$$

Proof. The notations of Theorem 7 are used here, as well. It follows from (22) and (23) that the components of the vector $2^{(j)}$ are the followings if $j<n$

$$
z_{i}^{j}=\frac{\beta_{j+1}}{\beta j}-1 \quad i=2, \ldots, j-1 ; \quad z_{j}^{(j)}=\cdots=z_{n}^{(j)}=0 .
$$

Here all components of $z^{(j)}$ are as great as possible. Therefore $\mathbf{z}^{(j)}=\mathbf{w}^{(j)}$ when $j<n$. Thus the statement follows from Theorem 7 and the definition of $\psi$.

Formula (27) has been stated already in [Boros 87] under slightly different conditions and without recognizing its connection to the greedy method.

Theorems 11 and 12 give a general method for solving problems with fixed $\alpha_{j}, \beta_{j} \quad(j=2, \ldots, n)$ and variable $a_{1}$. If $a_{1}$ is great enough, then (26) holds and the Frobenius number is obtained from Theorem 11 . This is illustrated by the following rather general example.

THEOREM 13. Assume that

$$
\alpha_{2}=\cdots=\alpha_{n}=\alpha
$$

and the numbers

$$
\frac{\beta_{j+1}}{\beta_{j}} \quad j=2, \ldots, n-1
$$

are integers and the positive integers $c$ and $v$ are defined by the constraints

$$
a_{1}=c \beta_{n}+v, \quad 1 \leq v<\beta_{n} .
$$

Let $\mathrm{t}$ be the greedy solution of $\left(K\left(v \mid x_{1}=0\right)\right)$. Finally let

$$
P=\left\{i: t_{i}<\frac{\beta_{i+1}}{\beta i}-1,2 \leq i<n\right\}
$$

and

$$
p= \begin{cases}1 & \text { if } P=\emptyset \\ \max \{i \in P\} & \text { if } P \neq \emptyset\end{cases}
$$

If

$$
\sum_{j=2}^{n} \frac{\beta_{j+1}}{\beta j} \leq \begin{cases}c+n & \text { if } \alpha=v=1 \\ c+n-1 & \text { if } \alpha>1 \text { and } v=1 \\ c+n+1 & \text { if } \alpha=1 \text { and } v>1 \\ c+n & \text { if } \alpha, v>1\end{cases}
$$


then the Frobenius number is

$$
a_{1} \alpha\left(\sum_{j=2}^{n-1} \frac{\beta_{j+1}}{\beta j} c-n\right)+c \beta_{n}+\beta_{n}-\beta_{p+1}-1
$$

Proof. At first the equation

$$
r_{n+1}=c \beta_{n}+\beta_{n}-\beta_{p+1}-1
$$

will be proven. As in the appropriate knapsack problem the coefficients of the objective function are equal, therefore $r_{n+1}$ is the integer between 1 and $a_{1}-1$, such that in the appropriate greedy solution the sum of the components is maximal. Not all components can achieve the maximal possible value, i.e. the number $\frac{\beta_{i+1}}{\beta i}-1$. The maximal sum is certainly achieved if all of the components have the maximal value, except one which is less by 1 . This upper bound of the sum is

$$
c+\sum_{j=2}^{n-1}\left(\frac{\beta_{j+1}}{\beta j}-1\right)-1=c+\sum_{j=2}^{n-1} \frac{\beta_{j+1}}{\beta_{j}}-n+1
$$

Let $x$ be the vector, where $x_{n}=c, x_{p+1}=\frac{\beta_{R+2}}{\beta p+1}-2$ and $x_{j+1}=\frac{\beta_{j+1}}{\beta j}-1(j \neq p+1, n)$, where $x_{n}=c-1$ if $p=n-1$. Then

$$
\sum_{j=2}^{n} x_{j} \beta_{j}=c+\sum_{j=2}^{n-1}\left(\frac{\beta_{j+1}}{\beta j}-1\right) \beta_{j}-\beta_{p+1}=c \beta_{n}+\beta_{n}-\beta_{p+1}-1
$$

It follows from the definition of $p$, that this is the greatest such sum being less than $a_{1}$. This implies (30). Hence

$$
h_{n+1}=\alpha\left(c-n+1+\sum_{j=2}^{n-1} \frac{\beta_{j+1}}{\beta j}\right) .
$$

The last item to prove is the inequality (27). Now $h\left(r_{n+1}+k a_{1}\right)$ is at least

$$
\begin{aligned}
\alpha\left\lceil\frac{r_{n+1}+k a_{1}}{\beta n}\right\rceil & =\alpha\left\lceil\frac{c \beta_{n}+\beta_{n}-\beta_{p+1}-1+k c \beta_{n}+k v}{\beta n}\right\rceil= \\
& =\alpha(k+1) c+\alpha\left\lceil\frac{\beta_{n}-\beta_{p+1}-1+k v}{\beta n}\right\rceil .
\end{aligned}
$$

In the last term the numerator is a nonnegative integer and can be zero only if $v=1$. Thus the right-hand side of (27) is at least $\alpha(k+1) c+k$ if $v=1$ and $\alpha(k+1) c+\alpha+k$ otherwise. Hence (27) follows from (29).

[Dulmage-Mendelsohn 64] discussed two special cases of this theorem. One of them is given in the following corollary. The formula of the Frobenius number is simplified. 
COROLlaRY 1. Using the notations of the last theorem, assume that $n=4$ and $a_{1} \geq 2$ and $a_{2}=a_{1}+1, a_{2}=a_{1}+2, a_{2}=a_{1}+6$. Then the Frobenius number is

$$
\left(a_{1}+6\right)(c+1)-\delta
$$

where $\delta=7$ if $v=0,1,2,3$ and $\delta=3$ if $v=4$ and $\delta=2$ if $v=5$.

Proof. The formula immediately follows from Theorem 10 and 13. But (29) claims that $a_{1}>1$.

Indeed, the formula gives 0 if $a_{1}=1$ instead of the correct value, which is -1 . This was not noticed in [Dulmage-Mendelsohn 64].

Another special case has been discussed in [Selmer 77]. This is the generalization of the other case solved by [Dulmage-Mendelsohn 64].

CoRollary 2. Using the notation of the last theorem, assume that

$$
a_{1} \geq(n-5) 2^{n-2}+2
$$

and $a_{j}=a_{1}+2^{j-2}, j=2, \ldots, n$. Let $b_{n-2} b_{n-3} \ldots b_{1} b_{0}$ the binary form of $v$ and

$$
p=\left\{\begin{array}{l}
-1 \text { if }\left\{j: b_{j}=0\right\}=\emptyset \\
\max \left\{j: b_{j}=0\right\} \text { otherwise }
\end{array}\right.
$$

$p=\max \left\{j: b_{j}=0\right\}$. Then the Frobenius number is

$$
a_{1}\left(n-4+\left\lfloor\left\{\frac{a_{1}}{2^{n-2}}\right\rfloor\right)+2^{n-2}\left(\left\lfloor\frac{a_{1}}{2^{n-2}}\right\rfloor\right)-2^{p+1}-1 .\right.
$$

Proof. As $\alpha=1$ therefore the current form of (29) is (32).

\section{Extending the results}

In this section a method is developed to drop Constraint (8) in some cases. Let us consider a Frobenius problem with coefficients

$$
a_{1}=a, \quad a_{j}=\alpha_{j} a+\beta_{j} \quad(j=2, \ldots, n),
$$

where $\alpha_{j}, \beta_{j}(j=2, \ldots, n)$ are positive integers defined in (5). This problem will be called the first problem. A second problem can be obtained from it by the following transformation. Let $b$ be a fixed positive integer, such that $\operatorname{gcd}(a, b)=1$. Then the coefficients of the second problem are

$$
a_{1}^{\prime}=a, \quad a_{j}^{\prime}=\alpha_{j} a+\beta_{j} b \quad(j=2, \ldots, n) .
$$

Note that it is not assumed that $\beta_{j} b<a(j=2, \ldots, n)$. The residue classes mod $a$ and their smallest representable elements, i.e. the numbers defined in (2), for the first problem and the second problem, resp., are denoted by $M_{1}, \ldots, M_{a-1}, t_{1}, \ldots$, $t_{a-1}$ and $M^{b}, \ldots, M^{(a-1) b}, t^{b}, \ldots, t^{(a-1) b}$, resp. The typical terms are denoted $M_{p}$, $t_{p}, M^{p b}, t^{p b}$, where it is understood that $p$ lies between 1 and $a-1$ inclusive. 
THEOREM 14. Let $M_{p}$ be an arbitrary residue class $\bmod a$ and $w \in M_{p} \cap F$ be an arbitrary element. If $\mathbf{x}$ is a representation of $w$, such that

$$
\sum_{j=2}^{n} \beta_{j} x_{j}=p+y a
$$

then $x$ is a representation of $w+(b-1)(p+y a) \in M^{p b} \cap F$. Conversely, if $v \in M^{p b} \cap F$ is an arbitrary element with representation $\mathrm{x}$, and

$$
\sum_{j=2}^{n} \beta_{j} x_{j}=p+y a
$$

then $\mathrm{x}$ is a representation of $v+(1-b)(p+y a) \in M_{p} \cap F$.

Proof. It is known from the conditions that

$$
w=\sum_{j=2}^{n} a_{j} x_{j}=a \sum_{j=2}^{n} \alpha_{j} x_{j}+\sum_{j=2}^{n} \beta_{j} x_{j}=a \sum_{j=2}^{n} \alpha_{j} x_{j}+y a+p .
$$

Hence

$$
\sum_{j=2}^{n} a_{j}^{\prime} x_{j}=a \sum_{j=2}^{n} \alpha_{j} x_{j}+b y a+b p=w+(b-1)(p+y a) .
$$

The proof of the opposite direction is similar.

TheOREM 15. Let $M_{p}$ be an arbitrary residue class. Let $\mathbf{x}$ be a representation of $t_{p}$. Furthermore let

$$
s=\sum_{j=2}^{n} \alpha_{j} x_{j} \text { and } p+y a=\sum_{j=2}^{n} \beta_{j} x_{j} .
$$

then the following two statements are equivalent:

(i) $t^{p b}<t_{p}+(b-1)(p+y a)$,

(ii) there is an element $t \in M_{p} \cap F$ with a representation $z$, such that if

$$
r=\sum_{j=2}^{n} \alpha_{j} x_{j} \text { and } p+w a=\sum_{j=2}^{n} \beta_{j} z_{j} .
$$

then the inequality

$$
b(w-y)<s-r
$$

holds.

Proof. Let $z$ be a representation of $t^{p b}$. Let the integers $r$ and $w$ defined by the equations

$$
r=\sum_{j=2}^{n} \alpha_{j} x_{j} \text { and } p b+w a b=\sum_{j=2}^{n} \beta_{j} b z_{j}
$$


The statement (i) is true if and only if

$$
a r+b p+a b w<a s+b p+a b y .
$$

Then (ii) holds with

$$
t=\sum_{j=2}^{n} a_{j} z_{j}=a(r+w)+p \in M_{p} .
$$

Conversely assume that an element $t \in M_{p}$ exists, such that (35) holds. Then let

$$
t^{\prime}=t+(b-1)(p+w a)=a(r+b w)+b p \in M^{p b} \cap F
$$

and

$$
t^{\prime \prime}=t_{p}+(b-1)(p+y a)=a(s+b y)+b p \in M^{p b} \cap F .
$$

Then it follows from (36) that $t^{\prime}<t^{\prime \prime}$.

THEOREM 16. Let $M_{p}$ be an arbitrary residue class. Let $\mathbf{x}$ be a representation of $t_{p}$. Furthermore let $s$ and $y$ be the integers defined in (34). If $y=0$ and $b>1$ then

$$
t^{p b}=t_{p}+(b-1) p
$$

Proof. Let $t \in M_{p} \cap F$. Furthermore let $r$ and $w$ be the integers defined in (35). It follows from the definition of $s$ and $y$ that

$$
s=s+y \leq r+w .
$$

If $w=0$ then $r \geq s$ and thus (36) cannot hold. Otherwise assume that (36) holds. Then the following sequence of relation is obtained from (36)

$$
s>b w+r=(b-1) w+w+r \geq(b-1) w+s>s,
$$

which is a contradiction.

Notice that in all of the exactly solved cases the condition $y=0$ has been satisfied for $r_{n+1}$, this the last theorem gives an immediate extension of the results of the previous section.

\section{REFERENCES}

[Boros 87] E. Boros, On a linear diophantine problem for geometrical type sequences, Discrete Math 66 (1987), 27-33.

[Brauer-Shockley 62] A. Brauer, J. E. Shockley, On a Problem of Frobenius, $J$. reine angew. Math., 211 (1962), 215-220.

[BYrnes 74] J.S. BYRnes, On a Partition Problem of Frobenius, J. Combinatorial Theory, Ser. A, 17 (1974), 162-166.

[Dulmage-Mendelsohn 64] A. L. Dulmage, N. S. Mendelsohn, Gaps in the Exponent Set of Primitive Matrices, Mlinois J. Math., 8 (1964), 642-656. 
[Hujter 82] M. Hujter, On a Sharp Upper and a Lower Bound for the Frobenius Problem, Computer and Automation Institute of the Hungarian Academy of Sciences, Report No. MO/32.

[Johnson-Kernighan Xx] S. C. Johnson and B. W. Keringhan, Making change with a minimum number of coins, manuscript, undated, Bell Laboratories, Murray Hill, New Jersey.

[KanNan 92] R. KanNaN, Lattice translates of polytope and the Frobenius problem, Combinatorica 12 (1992), 161-177.

[Magazine et al. 75] M. J. Magazine, G. L. Nemhauser, L. E. Trottler, When the greedy solution solves a class of knapsack problems, Operations Research 23 (1975), 207-217.

[Roberts 56] J. B. Roberts, Note on the Linear Forms, Proc. Amer. Math. Soc., 7 (1956), 465-469.

[SELMer 77] E. Selmer, On the linear diophantine problem of Frobenius, J. reine angew. Math. 293/294 (1977), 1-17.

[SiERING 74] E. Siering, Über lineare Formen und ein Problem von Frobenius, J. reine angew. Math. 271 (1974), 177-202.

[VIZVÁRI 77] B. VIZVÁRI, On the optimal solutions of discrete programming algorithms (in Hungarian), Alkalmazott Matemetikai Lapok, 3 (1977), 139-150.

[Vizvári 84] B. Vizvári, On the connection of the Frobenius Problem and the Knapsack Problem, in Colloquia Mathematica Societatis János Bolyai, 37. Finite and Infinite Sets, North-Holland 1984, 799-819.

[VIZVÁRI 87] B. VIZVÁRI, An application of Gomory Cuts in Number Theory, Periodica Mathematica Hungarica 18 (1987), 213-228.

[VIZVÁRI] B. VIZVÁRI, On the Optimality of the Greedy Solutions of the General Knapsack Problem, Optimization, 23 (1992), 125-138.

(Received May 1, 1992)

BILKENT UNIVERSITY

06533 BILKENT

ANKARA

TURKEY

E-MAIL: VIZVÁRI@TRBILUN.BITNET 